\title{
Challenges in Integrative Research in Therapeutic and Diagnostic Proteins: Translation and Conformational Engineering
}

\author{
Ajay K Ray* \\ Department of Chemical and Biochemical Engineering, University of Western Ontario, Canada
}

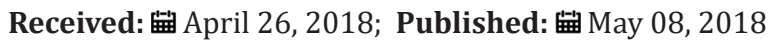

*Corresponding author: Ajay K Ray, Department of Chemical and Biochemical Engineering, University of Western Ontario, Canada, Email: aray6@uwo.ca

\begin{abstract}
Abbreviations: MAbs: Monoclonal Antibodies; CHO: Chinese Hamster Ovary; MCCSMB: Multiple Column Chromatography Based on Simulated Moving Bed Technology
\end{abstract}

\section{Mini Review}

Biopharmaceuticals are mostly protein-derived medical drugs play vital roles in the human body catalyzing sequence of biochemical reactions. These biologics, which include therapeutic proteins, monoclonal antibodies (MAbs), vaccines, hormones, and fusion proteins, are used in the treatment, diagnosis and prevention of specific diseases such as cancer, multiple sclerosis, rheumatoid arthritis, diabetes, and a variety of cardiovascular diseases [1-3]. They are large molecular weight compounds with complex 3D structures mimicking molecules found in human bodies. Their production by living cells makes them different from classical small molecular weight chemical drugs (e.g., antibiotics) and in general, protein drug production by living cells is generally difficult [3]. With the advent of recombinant DNA technology, the post genomic era is seeing a massive increase in valuable protein products entering the clinical trials to manage disease with high specificity [4]. The global market for recombinant bio-pharmaceuticals has been growing rapidly from 30 products with a market value of USD \$50-60 billion in 2004 to more than 151 unique products approved by the FDA by 2012 valued at USD $\$ 138$ billion and is expected to surpass $\$ 320$ billion by 2020 .

Manufacturing of the biopharmaceuticals involves drug discovery (identification of genetic code), expression of the desired product in the host cell to transcribe and translate the DNA's message into the biological medicine (upstream), stable cell line production where grown cells are cultured in bioreactors (midstream), recovery and purification of active and pure product (downstream), and drug formulation [5]. These stages are integrated, and therefore, be developed in tandem as downstream operations (currently account for over $50-70 \%$ of the production cost) are greatly impacted by the presence of inactive proteins as well as impurities [6]. About $50 \%$ of the recombinant protein therapeutics is expressed in E. coli (most economical choice for expression of nonglycosylated proteins) due to its well-characterized genetics, rapid growth rate, high yield, high productivity and low production cost [1]. However, the primary challenge resulting from protein over expression in E. coli is the formation of insoluble inactive protein aggregates (inclusion bodies, IB) containing misfolded inactive proteins [7]. Restoration of the biological activity by refolding into its unique 3D 'native state' for effective therapeutics remains a significant challenge [8-10]. To avoid the protein misfolding puzzles, production in mammalian cells (e.g., Chinese Hamster Ovary (CHO), although costly, has gained importance in generation of perfectly suited proteins in which it is possible to perform gene amplification and post translational modifications (e.g. glycosylation) for complete biological activity, which are otherwise impossible in E. coli [11-13]. But, mammalian cell production leads to the generation of many impurities (akin soup) such as host cell proteins (HCPs) and DNA fragments [6]. To comply with strict purity requirements of the target protein (which otherwise can affect safety, efficacy, or even trigger adverse immune responses in patients) [12], development of challenging downstream chromatographic purification method is required that involves multi-component separation of molecules having similar adsorptive properties [6]. In recent years, the upstream process platform has advanced extensively, both on the cell line expression and bioreactor process fronts, leading to high product titers. This has shifted the production bottleneck to large scale downstream purification processing that involves chromatography based protein refolding and purification as well as difficult multicomponent separation, which otherwise is difficult to achieve. 
Most industrial bioprocesses are operated in batch mode, which is a slow low yield process requiring large amount of mobile phase and resulting in very dilute product volumes [14,15]. Multiple Column Chromatography based on Simulated Moving Bed technology (MCCSMB) is a large scale version of the traditional High Performance Liquid Chromatography (HPLC) that has the potential to address these bioprocessing challenges [16]. SMB is used to separate binary mixture that is difficult using traditional separation techniques ensuing high purity products. Unlike HPLC, SMB operates in continuous mode with solvent recycling. The SMB unit consists of a set of fixed-bed chromatographic columns connected in series and segmented into 4 zones by inlet/outlet lines. It is operated in a mixed discrete and continuous mode; the inlet/outlet lines are periodically shifted synchronously in the direction of fluid phase flow to mimic countercurrent movement between a liquid solvent and a solid adsorbent phase. The inherent process intricacy in SMB offers numerous advantages including up to 8-fold increase in productivity per unit mass of stationary phase and up to $80 \%$ lower solvent consumption compared to single-column traditional batch operation, and hence, less diluted products helping the product recovery step cheaper [16].

SMB processes are well established for many large-scale separations such as separation of xylene isomers [17], recovery of concentrated fructose syrup [18] and for chiral pharmaceutical products [20], however, barriers towards implementation into the bio-pharmaceutical industry still remain, particularly for protein refolding and its purification [19]. This is largely due to the perceived complexity in process configuration as well as ability to handle multi component mixtures [20-23]. To realize MCC as processing platform in the bio pharmaceutical industry several challenges related to finding suitable packing material, solvent systems, and bio molecule characteristics to meet regulatory constraints, as well as translation of the SMB process intricacy to superior bio processing capability, need to overcome. The inherent process complexity offers substantial advantages in terms of simultaneous increases in productivity, high product purity and reduced solvent requirements. Taking these advantages for bioprocessing requires fundamental understanding of continuous bio-chromatography using protein-based therapeutic biomolecules, which is the one of the key objectives applicant's research program [24-27] The limitation of SMB is the inability to achieve linear solvent gradients, or gradients other than step gradient as required in protein refolding and purification of products from a mixture of many compounds with very similar adsorptive properties.

In addition, the classical 4 zone SMB is restricted to binary separation. The new recent development in SMB technology is the use of gradients in solvent strength that alters the affinity of proteins towards the ion-exchange resins and the elution sequence36, and the concept of MCC to permit solvent gradients in each column paving for purification of more complex multi-component mixtures [26]. Different salt concentrations in the feed and eluent create regions of high and low affinity. The high affinity state allows high loading while low affinity site facilitates protein elution, thereby increasing throughput and reducing solvent consumption compared to isocratic condition. The solvent gradient MCC (SG-MCC) retains the advantages of SMB (productivity, purity, solvent consumption) using conventional ion-exchange resins thus eliminating the need of costly Protein an affinity materials. Due to mass transfer limitations and selectivity's among the antibody variants close to 1 , the realized yield is below $10 \%$ for a desired purity of above $80 \%$ unless solvent gradient is used. Our recent work [24-26] on lysozyme protein refolding and solvent gradients showed improved performance (high productivity and low buffer consumption). Based on recent contemporary literature, researchers need to generate new knowledge through fundamental studies to facilitate rational process design and scale-up methodologies of MCC-SMB aimed in enhancing productivity and purity, reducing solvent consumption, improving process economics and time to market.

The common approach used to retrieve active protein from inclusion bodies involves three steps: inclusion body isolation, solubilization of the aggregated protein, and refolding of the solubilized protein. The efficiency of the first two steps is relatively high but renaturation yield is limited by the accumulation of inactive misfolded species and aggregates [24]. Critical to the development of an efficient and economic denaturant based solubilization steps for full bioactivity recovery in the refolding are:

a) Determination of the minimum amount of denaturant (e.g., urea) needed.

b) Addition of a reducing agent (e.g., dithiothreitol, DTT) to maintain cysteine residues in the reduced state preventing non-native intra and inter disulfide bond formation in highly concentrated protein solutions at alkaline pH. After solubilization, renaturation is accomplished by the removal of excess denaturants either by dilution or by a buffer exchange step. The correct protein folding pathway competes with misfolding and protein aggregation that plays a key role for industrial implementation in the making of recombinant proteins. During protein refolding, the first order refolding reaction competes with higher-order aggregation reaction, which substantially reduces renaturation yields. The renaturation yields can be further enhanced by suppressing aggregation by adding additives such as L-arginine. The key to a commercially viable renaturation process lies in understanding of inclusion body solubilization methods, the role additives play in the inhibition of aggregation and optimization of all these steps to increase the overall yield. Fast refolding kinetics, high refolding yields and increased efficiency addressing technical challenges in large scale MCC SMB processes are critical in determining the speed with which new protein drugs can be brought to market.

Researchers need to work with solvent gradients along each column in SG MCC providing an extra degree of control that is inefficient in batch columns and to understand the effect of location 
and solvent gradient strengths, determination of Steric Mass Action isotherm, flow rates in different sections, switching time and column configuration for purification of antibodies. In MCC, by synchronous (as in SMB) and non-synchronous (as in Varicol) switching of the inlet and outlet ports, the relative velocities of the solid and the fluid phase are controlled which determines the extent of countercurrent movement and the ultimate process performance [28]. The target protein moves in the direction of the solid phase until it is refolded in the presence of refolding buffers and eluted with a suitable eluent.

Through appropriate setting of flow rates and setting suitable concentration gradients in different sections of MCC through variation of switch time and gradient concentrations; it is possible to achieve simultaneously purity and yield above 90\%. Different process schemes (column configurations) needs to be explored for continuous refolding of pure denatured protein containing weakly adsorbed contaminants, strongly adsorbed contaminants, and both weakly and strongly adsorbed contaminants along with the intermediate target protein. The new knowledge from fundamental study of different process schemes for multi component separation in SG MCC will address technical challenges that have caused MCC to be unexplored for large scale bio processing. New knowledge generated will facilitate optimum process design, innovative rational design and scale up methodology enhancing productivity of continuous protein bio processing, improving process economics, and opportunities for commercialization.

\section{References}

1. FR Schmidt (2004) Recombinant expression systems in the pharmaceutical industry. Applied Microbioogy Biotechnology 65(4): 363-372.

2. JR Birch, Y Onakunle (2005) Biopharmaceuticals proteins: Opportunities and challenges. Methods Molecular Biology 308: 1-16.

3. B Leader, QJ Baca, DE Golan (2008) Protein therapeutics: A summary and pharmacological classification. Nature Reviews 7(1): 21-39.

4. I Rosenfeld, Borin Van Loon, Edward Ziff (2010) DNA: A graphic guide to the molecule that shook the world. Columbia Univ Press, USA.

5. Shukla, J Thömmes (2010) Recent advances in large scale production of monoclonal antibodies and related proteins. Trends in Biotechnology 28(5): 253-261.

6. T Müller Spath, L Aumann, L Melter, G Ströhlen, M Morbidelli (2008) Chromatographic separation of monoclonal antibody variants, Biotechnology \& Bioengineering 100(6): 1166-1177.

7. C Huang, H Lin, X Yang (2012) Industrial production of recombinant therapeutics in E. coli and its recent advances. J Industrial Microbiology Biotechnology 39(3): 383-399.

8. AS Rosenberg (2000) Effects of protein aggregates: An immunologic perspective. AAPS J 8(3): 501-507.

9. D Baker (2000) A surprising simplicity to protein refolding. Nature 405(6782): 39-42.

10. CM Dobson (2003) Protein folding and misfolding. Nature 426(6968): 884-890.
11. D Bandaranayake, SC Almo (2014) Recent advances in mammalian protein production. Federation European Biochemistry Society Letters 588(2): 253-260.

12. L Yin, X Chen, P Vicini, Rup B, T Hickling (2015) Therapeutic outcomes, risk factors and mitigation efforts of immunogenicity of therapeutic protein products. Cell Immunology 295(2): 118-126.

13. C Zhang, D Fredericks, E Campi, C Schiødt, M Hearn, et al. (2015) Purification of monoclonal antibodies by chemical affinity mixed mode chromatography. Sep Purification Technol 142: 332-339.

14. R Ho, Gibaldi M (2013) Large scale production of recombinant proteins in biotechnology and biopharamaceuticals: Transforming proteins into drugs. John Wiley \& Sons Inc Hoboken, USA.

15.FM Wurm (2004) Production of recombinant protein therapeutics in cultivated mammalian cells. Nature Biotechnology 22(11): 1393-1398.

16. K Ray, RW Carr, R Aris (1994) Simulated Counter current Moving Bed Chromatographic Reactor: A Novel Reactor Separator. Chemical Engineering Science 49(4): 469-480

17. Kurup, K Hidajat, AK Ray (2005) Optimal operation of an industrial Parex process for the recovery of $\mathrm{p}$ xylene from a mixture of $\mathrm{C}_{8}$ aromatics. Ind Eng Chem Res 44(15): 5703-5714.

18. Y Zhang, K Hidajat, AK Ray (2007) Modified reactive SMB for production of concentrated Fructose syrup by isomerization of glucose to fructose. Biochemical Engineering Journal 35(3): 341-351.

19.S Maria, G Joucla, WM Lomenech, X Santarelli, C Cabanne, et al. (2015) Purification process of recombinant monoclonal antibodies with mixed mode chromatography. J Chromatography 1393: 57-64.

20. W Yu, K Hidajat, AK Ray (2003) Modeling, simulation and experimental study of the SMB Reactor for the Synthesis of Methyl Acetate Ester. Ind Eng Chem Res 42(26): 6743-6754.

21. P Kundu, Y Zhang, AK Ray (2009) Modelling and simulation of oxidative coupling of methane in a simulated moving reactor. Chemical Engineering Science 64(24): 5143-5152.

22.S Kurup, K Hidajat, AK Ray (2006) Optimal operation of a Pseudo SMB process for ternary separation under non-ideal conditions. Separation and Purification Technology 51(3): 387-403.

23. S Kurup, K Hidajat, AK Ray (2006) Comparative study of modified SMB at optimal conditions for the separation of ternary mixtures under non ideal conditions. Ind Eng Chem Res 45(11): 3902-3915.

24. P Saremirad, J Wood, Y Zhang, AK Ray (2014) Multi variable operational characteristic studies of on column oxidative protein refolding at high loading concen. J Chromatography A 1359: 70-75.

25. P Saremirad, J Wood, Y Zhang, AK Ray (2014) Oxidative protein refolding on SEC at high loading concern: Fundamentals and mathematical Modeling. J Chromatography A 1370: 147-155.

26. P Saremirad, J Wood, Y Zhang, AK Ray 138 (2015) Oxidative protein refolding on SEC: From batch single column to multi column continuous processing. Chem Eng Sci 138: 375-384.

27. N Faraji, Y Zhang, AK Ray (2015) Determination of adsorption isotherm parameters for minor whey proteins by gradient elution liquid chromatography. J Chromatography A 1412: 67-74.

28. Z Zhang, K Hidajat, M Morbidelli,AK Ray (2002) Multi objective optimization of simulated moving bed system and Varicol process for chiral separation. AIChE Journal 48(12): 2800-2816 


\section{CC) (P) This work is licensed under Creative}

To Submit Your Article Click Here:

Submit Article
DOI: $10.32474 /$ OAJBEB.2018.02.000138

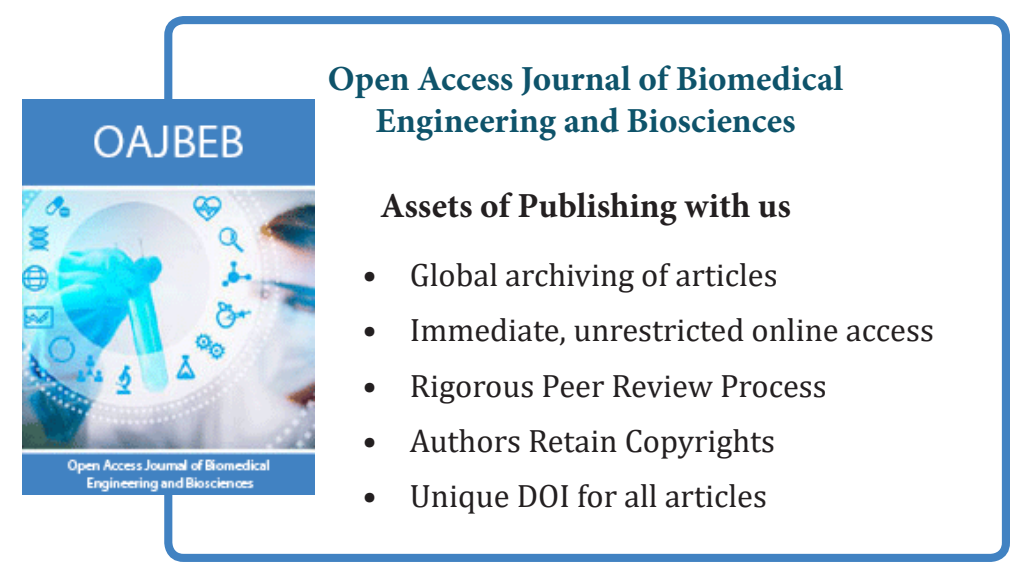

